\title{
Fuzzy Data Envelopment Analysis: A Discrete Approach
}

\author{
Majid Zerafat Angiz L. ${ }^{1}$ Ali Emrouznejad ${ }^{+2}$ Adli Mustafa ${ }^{3}$ \\ ${ }^{1}$ Department of Mathematics, Islamic Azad University, Firouzkooh, Iran \\ ${ }^{2}$ Aston Business School, Aston University, Birmingham, UK \\ ${ }^{3}$ School of Mathematical Sciences, Universiti Sains Malaysia, Penang, Malaysia
}

\begin{abstract}
Data envelopment analysis (DEA) as introduced by Charnes et al [3] is a linear programming technique that has widely been used to evaluate the relative efficiency of a set of homogenous decision making units (DMUs). In many real applications, the input-output variables cannot be precisely measured. This is particularly important in assessing efficiency of DMUs using DEA, since the efficiency score of inefficient DMUs are very sensitive to possible data errors. Hence, several approaches have been proposed to deal with imprecise data. Perhaps the most popular fuzzy DEA model is based on $\alpha$-cut. One drawback of the $\alpha$-cut approach is that it cannot include all information about uncertainty. This paper aims to introduce an alternative linear programming model that can include some uncertainty information from the intervals within the $\alpha$-cut approach. We introduce the concept of "local $\alpha$-level" to develop a multiobjective linear programming to measure the efficiency of DMUs under uncertainty. An example is given to illustrate the use of this method.
\end{abstract}

Keywords: Fuzzy data envelopment analysis; interval data; local $\alpha$-level, multi objective programming, decision making unit.

\section{Introduction}

Data envelopment analysis (DEA) initially was proposed by Charnes et al. [3] is a non-parametric linear programming technique for measuring the relative efficiency of a set of homogeneous decision making units (DMUs), with the common set of inputs and outputs. Examples of DEA include the efficiency of hospitals in providing their services [17] measurement efficiency of health

\footnotetext{
${ }^{\dagger}$ Corresponding author:

Ali Emrouznejad, Operations and Information Management Group, Aston Business School, Aston University, Birmingham, United Kingdom a.emrouznejad@aston.ac.uk
} 
centers [12] manufacturing efficiency [23, 24] productivity of OECD countries [5, 6 ,7]. For some computational calculation of DEA methods see Emrouznejad [8] and for a recent theoretical survey and full list of applications of DEA see Cook and Seiford [4] and Emrouznejad et al. [9].

Traditionally, all input/output values of DMUs are crisp data, hence, most of the previous studies dealt with precise data. Theoretically, DEA measures the efficiency of each DMU by finding the distance of the DMU to the best practice; therefore, the efficiency scores are very sensitive to the data. If there is an outlier, then the efficiency scores of many DMUs may change substantially. Therefore, a key to the success of the DEA is to measure all inputs outputs accurately. However, in real application of production process many complicated factors are involved that makes difficult to measure inputs and outputs precisely. This makes a case where we need to measure the efficiency of DMUs with inexact values or interval data.

Several approaches have been developed to deal with fuzzy data in DEA. Sengupta [26] applied principle of fuzzy set theory to introduce fuzziness in the objective function and the right-hand side vector of the conventional DEA model [3]. Guo and Tanaka [13] used the ranking method and introduced a bi-level programming model. Lertworasirikul [20] developed the method in which first, inputs and outputs were defazified and then the model was solved using the $\boldsymbol{\alpha}$ cut approach. Their method is simple but the uncertainty in inputs and outputs is effectively ignored. Some other approaches based on $\alpha$-cut can be found in [22, $16,25]$ and the methods based on the interval efficiency are seen in $[10,14]$.

Lertworasirikula [19] considered each constraint in the DEA as a fuzzy event; hence he transferred fuzzy DEA model to possibility linear programming problem. Lertworasirikul et al. [21] further developed a fuzzy BCC model where the possibility and credibility approaches are provided and compared with an $\alpha$-cut level based approach for solving the FDEA models. Using the possibility approach, they revealed the relationship between the primal and dual models of fuzzy BCC. Using the credibility approach they showed how the efficiency value for each $D M U$ can be obtained as a representative of its possible range. A different approach based on possibility programming was developed in [1, 18]. Inuiguchi and Tanino [15] applied the extension principle to define fuzzy efficiency score using DEA.

In this paper we develop an alternative method which is able to provide fuzzy efficiency measures for DMUs with fuzzy observations. We introduce the 
concept of local $\alpha$-level which can include more information from uncertain data into the model. Use of local $\alpha$-level in the proposed DEA model enables us to capture as much information as possible from the uncertain DMU while in the standard $\alpha$-cut some fuzzy characteristics of DMUs are ignored [27-29].

The rest of this paper is organized as follows. DEA and Fuzzy DEA are defined in Section 2. Using concept of local $\alpha$-level, an alternative fuzzy DEA is proposed in Section 3. Further discussion is given in Section 4. This is followed by a numerical example and comparison with other models in Section 5 . Conclusion is given in Section 6.

\section{DEA and fuzzy DEA}

Data envelopment analysis (DEA) is a non-parametric technique for measuring the relative efficiency of the decision making units (DMUs) that have homogenous inputs and outputs. DEA applies linear programming techniques to the observed inputs /outputs of DMUs by constructing an efficient production frontier based on the best practices. Each DMU's efficiency is then measured relative to its distance to this frontier [4].

Consider a set of $n$ DMUs, in which $x_{i j}(i=1,2, \ldots, m)$ and $y_{r j}(r=1,2, \ldots, s)$ are inputs and outputs of $D M U_{j}(j=1,2, \ldots, n)$. The standard form of CCR model for assessing $D M U_{p}$ is written as:

$$
\begin{array}{ll}
\max & \sum_{r=1}^{s} u_{r} y_{r p} \\
\text { s.t. } & \sum_{i=1}^{m} v_{i} x_{i p}=1 \\
& \sum_{r=1}^{s} u_{r} y_{r j}-\sum_{i=1}^{m} v_{i} x_{i j} \leq 0 \\
& u_{r}, v_{i} \geq \varepsilon \quad \forall r, i
\end{array}
$$

The above model can only be used for cases where the data are precisely measured. Fuzzy DEA is a powerful tool for evaluating the performance of DMUs with imprecise data (or interval data). Fuzzy input-output variables can be introduced to DEA in the following fuzzy linear programming model. 


$$
\begin{aligned}
& \max \sum_{r=1}^{s} u_{r_{\nu}} \sim \\
& \text { s.t. } \quad \sum_{i=1}^{m} v_{i^{*}}{ }^{*} \\
& \sum_{r=1}^{s} u_{r_{r}}, \frac{m}{i=1} \sim \\
& u_{r}, v_{i} \geq \varepsilon \quad \forall r, i
\end{aligned}
$$

where " $\sim$ "indicates the fuzziness. $\tilde{y}_{r j}$ and $\tilde{x}_{i j}$ are fuzzy inputs and fuzzy outputs, respectively. $\varepsilon \succ$ is a non-Archimedean small positive number.

Saati et al. [25] suggested a different CCR model for assessment of fuzzy data by transferring the standard CCR model to a possibilistic programming problem. Their basic idea is using $\alpha$-cut approach to transform the fuzzy CCR model (3) into a crisp linear programming problem such as the standard DEA model. Their proposed approach assumes that the solution lies in the interval and the result for each DMU is an interval efficiency score rather than a crisp efficiency score. The main drawback in this approach is that their model can not retain the uncertainty information completely since it is based on simple $\alpha$-cut approach. In other words, the fuzzy numbers are simply converted to intervals using the same membership numbers in the entire of interval.

\section{An alternative fuzzy DEA model under uncertainty}

The proposed approach in this paper is based on alternative definition of the membership functions of the coefficients, i.e. input output variables. Assume $F(\Re)$ is the set of fuzzy number. We define "local $\alpha$-level" as follows.

Definition 1: The crisp set of elements that belong to the fuzzy set $\tilde{M}$ with degree of at least $\alpha_{h}$ and less than $\alpha_{h+1}$, in which $\alpha_{h} \leq \alpha_{h+1}$, is called the "local $\alpha$ level" set and it is represented as follows:

$$
M_{\alpha_{h}}=\left\{x \in X \mid \alpha_{h} \leq \mu_{\tilde{M}}(x) \leftrightharpoons \alpha_{h+1}\right\}
$$

One advantage of using a local $\alpha$-level is that the corresponding point on the membership function retained the whole of uncertainty in process of solving the problem. We define the membership function $\mu_{\tilde{M} \tilde{N}}$ based on $\mu_{\tilde{M}}$ and $\mu_{\tilde{N}}$ [29]. 
Definition 2: If $\tilde{M}, \tilde{N}-F_{1} \ldots$, are two fuzzy numbers that $\mu_{\tilde{\Gamma}}(x) \mu_{\sim}(\ldots)$ are continuous membership functions, then

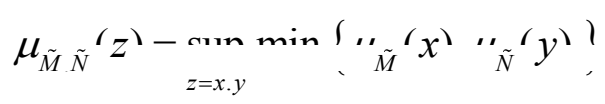

Obviously, if the bounded variable $v$ is considered as a trapezoidal fuzzy number, the following corollary is obtained.

Corollary 1: The product of bounded variable $v$ in fuzzy number $\tilde{M}$ is defined as

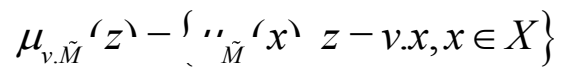

In most DEA applications for the sake of computational efficiency and ease of data acquisition, trapezoidal or triangular membership functions are often used.

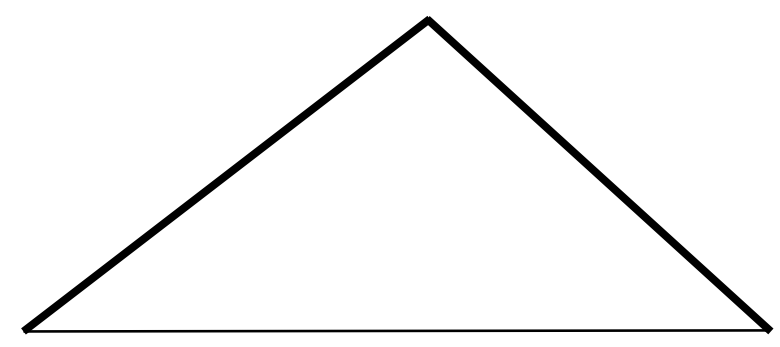

Figure 1a: Triangular membership function

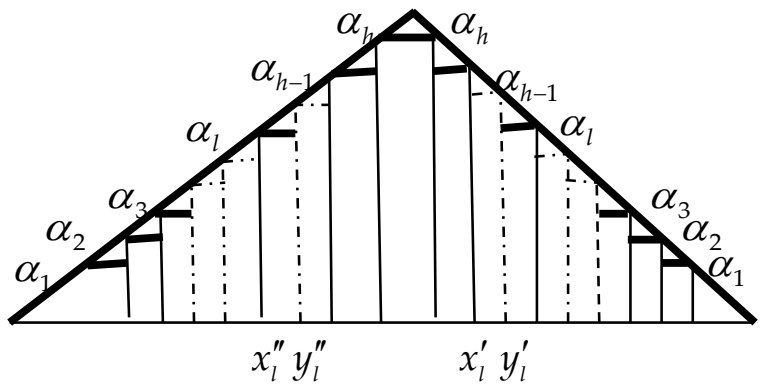

Figure 1b: concept of local $\alpha$-level

Figure 1a shows a triangular "fuzzy number" and Figure $1 \mathrm{~b}$ shows the local $\alpha$-level for a fuzzy number which is corresponding with the figure 1a. As seen, each $\alpha$-cut acts in a local determined domain. For instance, the corresponding domain of $\alpha_{l}$ is $\left[x_{l}^{\prime}, y_{l}^{\prime}\right] \cup\left[x_{l}^{\prime \prime}, y_{l}^{\prime \prime}\right]$. The range of each local $\alpha$-level in the figure $1 \mathrm{~b}$ has shown as the horizontal bold lines.

Theorem 1. Let $\tilde{A}=\left(x^{m}, x^{l}, x^{u}\right)$ be a symmetric triangular fuzzy number such that $\alpha_{i}(i=1,2, \ldots, n)$ are local $\alpha$-levels, thus, $x_{1}, x_{2}, \ldots, x_{n}, \ldots, x_{2 n}$ make a partition on the interval of fuzzy number $\tilde{A}$. Then, for each of the data point $x \in\left[x_{1}, x_{2 n}\right]$ we have

$$
\sum_{i=1}^{2 n} \mu_{\widetilde{A}}\left(x_{i}\right)\left|x_{i}-x^{m}\right| \leq \sum_{i=1}^{2 n} \mu_{\widetilde{A}}\left(x_{i}\right)\left|x_{i}-x\right|
$$

Proof. To prove this theorem, we rearrange (6) to 


$$
\sum_{i=1}^{2 n} \mu_{\widetilde{A}}\left(x_{i}\right)\left(\left|x_{i}-x^{m}\right|-\left|x_{i}-x\right|\right) \leq 0
$$

Suppose that

$$
B=\sum_{i=1}^{2 n} \mu_{\widetilde{A}}\left(x_{i}\right)\left(\left|x_{i}-x^{m}\right|-\left|x_{i}-x\right|\right)
$$

Two cases are considered

Case 1. $x_{k-1} \leq x \prec x_{k} \prec x^{m}$

In this case, B can be written in the following form.

$$
\begin{aligned}
B & =\sum_{i=1}^{k-1} \mu_{\widetilde{A}}\left(x_{i}\right)\left(\left(x^{m}-x_{i}\right)-\left(x-x_{i}\right)\right)+\sum_{i=k}^{n} \mu_{\widetilde{A}}\left(x_{i}\right)\left(\left(x^{m}-x_{i}\right)-\left(x_{i}-x\right)\right. \\
& +\sum_{i=n+1}^{2 n} \mu_{\widetilde{A}}\left(x_{i}\right)\left(\left(x_{i}-x^{m}\right)-\left(x_{i}-x\right)\right)
\end{aligned}
$$

Since $\widetilde{A}$ is symmetric, therefore $\mu_{\widetilde{A}}\left(x_{l}\right)=\mu_{\widetilde{A}}\left(x_{2 n-(l-1)}\right) \quad 1 \leq l \leq 2 n$. Thus, the right side of fuzzy number $\tilde{A}$ is divided to two areas as follows:

$$
\begin{aligned}
& \sum_{i=n+1}^{2 n} \mu_{\widetilde{A}}\left(x_{i}\right)\left(\left(x_{i}-x^{m}\right)-\left(x_{i}-x\right)\right)=\sum_{i=1}^{k-1} \mu_{\widetilde{A}}\left(x_{(2 n-(i-1))}\right)\left(\left(x_{(2 n-(i-1))}-x^{m}\right)-\left(x_{(2 n-(i-1))}-x\right)\right) \\
+ & \sum_{i=k}^{n} \mu_{\widetilde{A}}\left(x_{(2 n-(i-1))}\right)\left(\left(x_{(2 n-(i-1))}-x^{m}\right)-\left(x_{(2 n-(i-1))}-x\right)\right)
\end{aligned}
$$

Combining (8) and (9) we obtain

$$
\begin{aligned}
B & =\sum_{i=1}^{k-1} \mu_{\widetilde{A}}\left(x_{i}\right)\left[\left(\left(x^{m}-x_{i}\right)-\left(x-x_{i}\right)\right)+\left(\left(x_{(2 n-(i-1)}-x^{m}\right)-\left(x_{(2 n-(i-1)}-x\right)\right)\right] \\
& +\sum_{i=k}^{n} \mu_{\widetilde{A}}\left(x_{i}\right)\left[\left(\left(x^{m}-x_{i}\right)-\left(x_{i}-x\right)\right)+\left(\left(x_{(2 n-(i-1))}-x^{m}\right)-\left(x_{(2 n-(i-1)}-x\right)\right)\right]
\end{aligned}
$$

In equation (10) we have

$$
B=0+\sum_{i=k}^{n} \mu_{\widetilde{A}}\left(x_{i}\right)\left(-2 x_{i}+2 x\right)
$$


Since $x_{i} \succ x$ for $i \geq k$, therefore, $B \leq 0$.

Case 2. $x^{m} \leq x_{k-1} \leq x \prec x_{k} \leq 2 n$

Similarly, it can be proved that $B \leq 0$.

Hence, $\min _{x} \sum_{i=1}^{2 n} \mu_{\widetilde{A}}\left(x_{i}\right)\left|x_{i}-x\right|$ in this method is obtained the maximal degree of membership function which does not depend on number of partitions.

Corollary 2. Suppose $x_{i}, x, \mu_{\tilde{A}}\left(x_{i}\right)$ satisfy the conditions of Theorem 1 then $\min _{x} \sum_{i=1}^{2 n} \mu_{\widetilde{A}}\left(x_{i}\right)\left|x_{i}-x\right|$ is unique.

Using the concept of local $\alpha$-level in model (2) the following fuzzy linear programming is proposed for assessing $D M U_{p}$.

$$
\begin{aligned}
& \min Z_{i j}=\sum_{h} \mu_{\tilde{x}_{i j}}\left(x_{i j h}\right)\left|v_{i} \bar{x}_{i j}-v_{i} x_{i j h}\right| \quad \forall i, j \\
& \min Z_{r j}=\sum_{k} \mu_{\tilde{y}_{r j}}\left(y_{r j k}\right)\left|u_{r} \bar{y}_{r j}-u_{r} y_{r j k}\right| \quad \forall r, j \\
& \max Z_{2}=\sum_{r=1}^{s} u_{r} \bar{y}_{r p} \\
& \text { s.t. } \quad \\
& \qquad \sum_{i=1}^{m} v_{i} \bar{x}_{i p}=1 \\
& \quad \sum_{r=1}^{s} u_{r} \bar{y}_{r j}-\sum_{i=1}^{m} v_{i} \bar{x}_{i j} \leq 0 \quad \forall j \\
& x_{i j}^{l} \leq \bar{x}_{i j} \leq x_{i j}^{u} \quad \forall i, j \\
& y_{r j}^{l} \leq \bar{y}_{r j} \leq y_{r j}^{u} \quad \forall r, j \\
& u_{r}, v_{i} \geq \varepsilon \quad \forall r, i
\end{aligned}
$$

As seen in figure $1 \mathrm{~b}$, corresponding to each $\alpha$, a set of subintervals is assigned to each fuzzy number.

Theorem 2. The above model is an extension to proposed model of Kao and Liu [16], if we remove the two minimization distance functions from the objective of 
the model, the largest value of optimal solution in each $\alpha$-level is the same as those obtained in Kao and Liu [16].

Proof. Consider the following model:

$$
\begin{array}{ll}
\max & \sum_{r=1}^{s} u_{r} \bar{y}_{r p} \\
\text { s.t. } & \\
& \sum_{i=1}^{m} v_{i} \bar{x}_{i p}=1 \\
& \sum_{r=1}^{s} u_{r} \bar{y}_{r j}-\sum_{i=1}^{m} v_{i} \bar{x}_{i j} \leq 0 \\
\alpha y^{m}{ }_{r j}+(1-\alpha) y^{l}{ }_{r j} \leq \bar{y}_{r j} \leq \alpha y_{r j}^{m}+(1-\alpha) y_{r j}^{u} \\
\alpha x^{m}{ }_{i j}+(1-\alpha) x_{i j}^{l} \leq \bar{x}_{i j} \prec \quad(1-\alpha) x^{u}{ }_{i j} \\
u_{r}, v_{i} \geq 0 \quad \forall r, i
\end{array}
$$

For $\alpha=0$, consider $n D M U$ s with $m$ inputs and $r$ outputs as follows:

$$
\begin{array}{lll}
x_{i j}=x_{i j}^{u} \quad j \neq o & x_{i o}=x_{i p}^{l} \\
y_{r j}=y_{r j}^{l} & j \neq o & y_{r o}=y_{r p}^{u}
\end{array}
$$

It is clear that for $D M U_{o}$ each dominated DMU (a DMU with higher level of inputs and lower level of outputs) will not be more efficient. So optimal value of mathematical programming (12) is equals to efficiency of $D M U_{p}$. This is also valid for any arbitrary $\alpha$.

In model (11), $x_{i j h}$ corresponds to the length of input value of $x_{i j}$ located in the intersection of $\alpha_{h}$ and sides of the corresponding triangular membership function. Similarly $y_{r j k}$ corresponds to the length of output value of $y_{i j .}$. Consider the following variable substitutions.

$$
\hat{x}_{i j}=v_{i} \bar{x}_{i j}, \hat{y}_{r j}=u_{r} \bar{y}_{r j} \quad \forall i, r, j
$$


Hence, model (13) is concluded.

$$
\begin{aligned}
& \min Z_{i j}=\sum_{h} \mu_{\tilde{x}_{i j}}\left(x_{i j h}\right)\left|\hat{x}_{i j}-v_{i} x_{i j h}\right| \quad \forall i, j \\
& \min Z_{r j}=\sum_{k} \mu_{\tilde{y}_{r j}}\left(y_{r j k}\right)\left|\hat{y}_{r j}-u_{r} y_{r j k}\right| \quad \forall r, j \\
& \max Z_{p}=\sum_{r=1}^{s} \hat{y}_{r p} \\
& \text { s.t. } \quad \\
& \quad \sum_{i=1}^{m} \hat{x}_{i p}=1 \\
& \sum_{r=1}^{s} \hat{y}_{r j}-\sum_{i=1}^{m} \hat{x}_{i j} \leq 0 \quad \forall j \\
& v_{i} x_{i j}^{l} \leq \hat{x}_{i j} \leq v_{i} x_{i j}^{u} \quad \forall i, j \\
& u_{r} y_{r j}^{l} \leq \hat{y}_{r j} \leq u_{r} y_{r j}^{u} \quad \forall r, j \\
& u_{r}, v_{i} \geq \varepsilon \quad \forall r, i \quad
\end{aligned}
$$

Assume that $x_{i j h}^{\prime}=\hat{x}_{i j}-v_{i} x_{i j h},(\forall i, j, h)$, so $\left|x_{i j h}^{\prime}\right|=x_{i j h}^{+}+x_{i j h}^{-}(\forall i, j, h)$ and $y_{r j k}^{\prime}=\hat{y}_{r j}-u_{r} y_{r j k}$, so $\left|y_{r j k}^{\prime}\right|=y_{r j k}^{+}+y_{r j k}^{-}(\forall r, j, k)$. Applying these substitutions model (13) may be solved using the following multi-objective programming.

$$
\begin{array}{ll}
\min Z_{i j}=\sum_{h} \mu_{\tilde{x}_{i j}}\left(x_{i j h}\right)\left(x_{i j h}^{+}+x_{i j h}^{-}\right) & \forall i, j \\
\min Z_{r j}=\sum_{k} \mu_{\tilde{y}_{r j}}\left(y_{r j k}\right)\left(y_{r j k}^{+}+y_{r j k}^{-}\right) & \forall r, j \\
\max Z_{p}=\sum_{r=1}^{s} \hat{y}_{r p} &
\end{array}
$$

s.t.

$$
\begin{array}{cl}
\sum_{i=1}^{m} \hat{x}_{i p}=1 & \\
\sum_{r=1}^{s} \hat{y}_{r j}-\sum_{i=1}^{m} \hat{x}_{i j} \leq 0 & \forall j \\
\hat{x}_{i j}-v_{i} x_{i j h}-\left(x_{i j h}^{+}-x_{i j h}^{-}\right)=0 & \forall i, j, h \\
\hat{y}_{r j}-u_{r} y_{r j k}-\left(y_{r j k}^{+}-y_{r j k}^{-}\right)=0 & \forall r, j, k \\
v_{i} x_{i j}^{l} \leq \hat{x}_{i j} \leq v_{i} x_{i j}^{u} & \forall i, j \\
u_{r} y_{r j}^{l} \leq \hat{y}_{r j} \leq u_{r} y_{r j}^{u} & \forall r, j \\
u_{r}, v_{i} \geq \varepsilon \quad \forall r, i &
\end{array}
$$


The model (14) is a multi-objective, hence, it can be solved by Archimedean goal programming model (15) as follows:

$$
\begin{array}{lll}
\min & \sum_{i=1}^{m} \sum_{j=1}^{n} w_{i j}^{\prime} d_{i j}^{\prime}+\sum_{r=1}^{s} \sum_{j=1}^{n} w_{r j}^{\prime \prime} d_{r j}^{\prime \prime}+w_{p} d_{p} \\
\text { s.t. } & \\
& \sum_{h} \mu_{\tilde{x}_{i j}}\left(x_{i j h}\right)\left(x_{i j h}^{+}+x_{i j h}^{-}\right)-d_{i j}^{\prime} \leq t_{i j}^{\prime} \quad \forall i, j \\
& \sum_{k} \mu_{\tilde{y}_{r j}}\left(y_{r j k}\right)\left(y_{r j k}^{+}+y_{r j k}^{-}\right)-d_{r j}^{\prime \prime} \leq t_{r j}^{\prime \prime} \quad \forall r, j \\
& \sum_{r=1}^{s} \hat{y}_{r p}+d_{p} \geq t_{p} \\
& \\
\sum_{i=1}^{m} \hat{x}_{i p}=1 & \\
\sum_{r=1}^{s} \hat{y}_{r j}-\sum_{i=1}^{m} \hat{x}_{i j} \leq 0 & \forall j \\
\hat{x}_{i j}-v_{i} x_{i j h}-\left(x_{i j h}^{+}-x_{i j h}^{-}\right)=0 & \forall i, j, h \\
\hat{y}_{r j}-u_{r} y_{r j k}-\left(y_{r j k}^{+}-y_{r j k}^{-}\right)=0 & \forall r, j, k \\
v_{i} x_{i j}^{l} \leq \hat{x}_{i j} \leq v_{i} x_{i j}^{u} & \forall i, j \\
u_{r} y_{r j}^{l} \leq \hat{y}_{r j} \leq u_{r} y_{r j}^{u} \quad \forall r, j \\
u_{r}, v_{i} \geq \varepsilon \quad \forall r, i
\end{array}
$$

In model (15), the $w^{\prime}$ s in the objective function are positive penalty weights and $d$ 's measure the over-achievement and under-achievement from the target point $t$, i.e. $t_{i j}^{\prime}, t_{r j}^{\prime \prime}, t_{p}$.

\section{Discussion}

In Figure 2a, consider the local $\alpha$-level $\alpha_{1}$. The membership values corresponding to interval $\left[n_{1}, n_{2}\right]$ are approximated by $\alpha_{1}$. For instance, the membership value related to $x$ is $\alpha_{1}$ instead of $\mu(x)$ that is real membership value. Furthermore, assume that we include another local $\alpha$-level $\alpha_{2}$ (See Figure $2 b)$, it is seen that the membership function corresponding to $x$ is now $\alpha_{2}$ 
instead of $\mu(x)$. It is clear that $\alpha_{1} \prec{ }_{2} \prec(x)$ and $\alpha_{2}$ is a better approximation than $\alpha_{1}$ for $\mu(x)$.
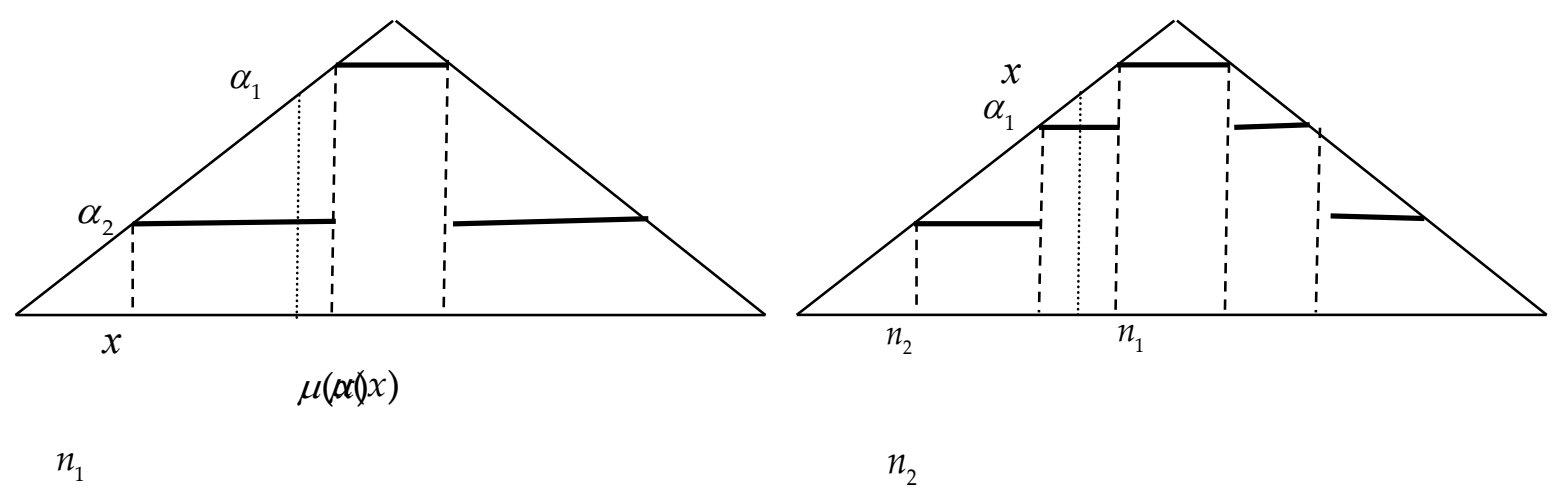

According to Theorems 2, if the objective functions minimizing in (13) are deleted from the model, the optimal solution for inputs and outputs will be arisen at its endpoints of interval of fuzzy numbers. Furthermore, if the objective function maximizing in (13) is eliminated, Theorem 1 is adopted and its optimal solutions are fuzzy number. Figure 3 illustrates the above mentioned concept for evaluating $D M U_{P}$. In this figure, the interior arrows represent the optimal solution when the objective function of maximizing is absent in (13) and the arrows located under fuzzy numbers construct the optimal solution (13) when only objective function of maximizing is present.
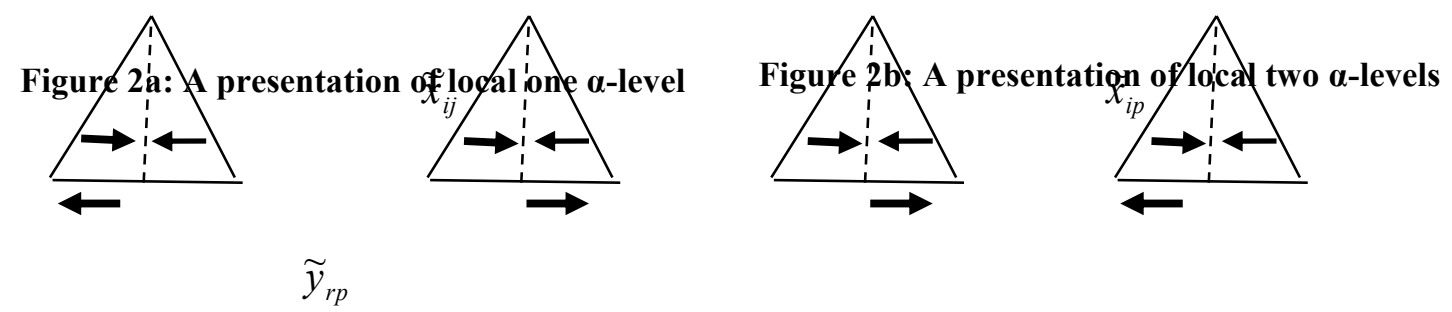

Interaction between the objective functions of maximizing and minimizing in (13) cause the fuzzy optimal solution.

In the methods based on $\alpha$-level approach, all fuzzy numbers are dealt with the same level. Consider the example given in Kao and Liu [16] evaluating four DMUs with single input and single output. In total, 88 LPs should be solved using the $\alpha$-cuts of the efficiency scores for eleven $\alpha$ values, they assumed that all fuzzy inputs and outputs are in the same $\alpha$-level. In the proposed method different level of $\alpha$ 's are considered for inputs and outputs, hence we need to solve $11 \times 11 \times 88$ LPs. 


\section{An Application and comparison with other methods}

Consider 5 DMUs with two triangular fuzzy inputs and 2 triangular fuzzy outputs. Table (1) shows the data which are also used in Guo and Tanaka [13].

Table 1. Data for numerical example

\begin{tabular}{clllll}
\hline $\begin{array}{c}\text { DMU } \rightarrow \\
\text { Variable }\end{array}$ & D1 & D2 & D3 & D4 & D5 \\
\hline I1 & $(4.0,3.5,4.5)$ & $(2.9,2.9,2.9)$ & $(4.9,4.4,5.4)$ & $(4.1,3.4,4.8)$ & $(6.5,5.9,7.1)$ \\
I2 & $(2.1,1.9,2.3)$ & $(1.5,1.4,1.6)$ & $(2.6,2.2,3.0)$ & $(2.3,2.2,2.4)$ & $(4.1,3.6,4.6)$ \\
O1 & $(2.6,2.4,2.8)$ & $(2.2,2.2,2.2)$ & $(3.2,2.7,3.7)$ & $(2.9,2.5,3.3)$ & $(5.1,4.4,5.8)$ \\
O2 & $(4.1,3.8,4.4)$ & $(3.5,3.3,3.7)$ & $(5.1,4.3,5.9)$ & $(5.7,5.5,5.9)$ & $(7.4,6.5,8.3)$ \\
\hline
\end{tabular}

Source: Guo and Tanaka (2001)

Fuzzy efficiencies of DMUs using standard fuzzy DEA model (2) and with different $\alpha$ value solved by the method suggested in [24] is reported in Table (2).

Table 2.the results of the model suggested in [24]

\begin{tabular}{|c|c|c|c|c|c|}
\hline $\begin{array}{c}\mathrm{DMU} \rightarrow \\
\alpha\end{array}$ & D1 & D2 & D3 & D4 & D5 \\
\hline 0 & 1.0 & 1.0 & 1.0 & 1.0 & 1.0 \\
\hline .5 & 0.995 & 1.0 & 1.0 & 1.0 & 1.0 \\
\hline .75 & 0.906 & 1.0 & 0.936 & 1.0 & 1.0 \\
\hline 1 & 0.85 & 1.0 & .86 & 1.0 & 1.0 \\
\hline
\end{tabular}

Using the Kao and Liu's approach, the $\alpha$-cut of the efficiency scores for four $\alpha$ values is presented in Table (3).

Table 3. Table of efficiency using Kao \& Liu fuzzy DEA model

\begin{tabular}{cccccc}
\hline DMU $\rightarrow$ & D1 & D2 & D3 & D4 & D5 \\
$\alpha$ & & & & & $(0.855,1.0)$ \\
\hline 0 & $(0.626,1.0)$ & $(0.835,1.0)$ & $(0.575,1.0)$ & $(10,1.0)$ & $(0.845,1.0)$ \\
.5 & $(0.757,0.995)$ & $(0.988,1.0)$ & $(10,1.0)$ & $(1.0,1.0)$ & $(0.971,1.0)$ \\
.75 & $(0.808,0.906)$ & $(1.0,1.0)$ & $(0.792,0.936)$ & 1.0 & 1.0 \\
1 & 0.85 & 1.0 & .86 & \\
\hline
\end{tabular}

The results of the possibility approach introduced in [19] have given in Table (4).

Table 4. Table of efficiency using_Lertworasirikul's possibilistic model [19]

\begin{tabular}{cccccc} 
DMU $\rightarrow$ & D1 & D2 & D3 & D4 & D5 \\
$\alpha$ & & & & & 1.296 \\
0 & 1.107 & 1.238 & 1.276 & 1.52 & 1.226 \\
0.25 & 1.032 & 1.173 & 1.149 & 1.386 & 1.159 \\
0.5 & 0.963 & 1.112 & 1.035 & 1.258 & 1.095 \\
0.75 & 0.904 & 1.055 & 0.932 & 1.131 & 1.000 \\
1 & 0.855 & 1.000 & 0.861 & 1.000 & \\
\hline
\end{tabular}


Although the Lertworasirikul's possibilistic model is not directly comparable with the new suggested method, the result of Table (4) is aggregated measure in a linear approach and an optimal $\alpha-c u t$ is obtained for each fuzzy numbesults to make it comparable.

Fuzzy efficiencies of $D M U$ s using the proposed model and with different $\alpha$ values are reported in Table (5).

Table 5. Table of efficiency using proposed fuzzy DEA model

\begin{tabular}{cccccc}
$\alpha$ & D1 & D2 & D3 & D4 & D5 \\
\hline $0,0.25,0.5,0.6,0,7,0.75,1$ & 0.915 & 1.0 & 0.948 & 1.0 & 0.991 \\
$0,0.25,0.5,0.7,0.75,1$ & 0.909 & 1.0 & 0.945 & 1.0 & 0.991 \\
$0,0.5,0.75,1$ & 0.903 & 1.0 & 0.941 & 1.0 & 0.84 \\
0 & 1.0 & 1.0 & 1.0 & 1.0 & 1.0 \\
\hline
\end{tabular}

The results shown in the first row of Table 4 are more accurate than the results suggested in [24], as seen in Theorem 2, Kao and Liu [16] model obtained the results at endpoints of the intervals only.

Figure 4 shows the structure of the discrete triangular number concerned to input 1 of $D M U_{1}$. As it can be seen we included 7 local $\alpha$-levels for calculation of efficiency in this example.

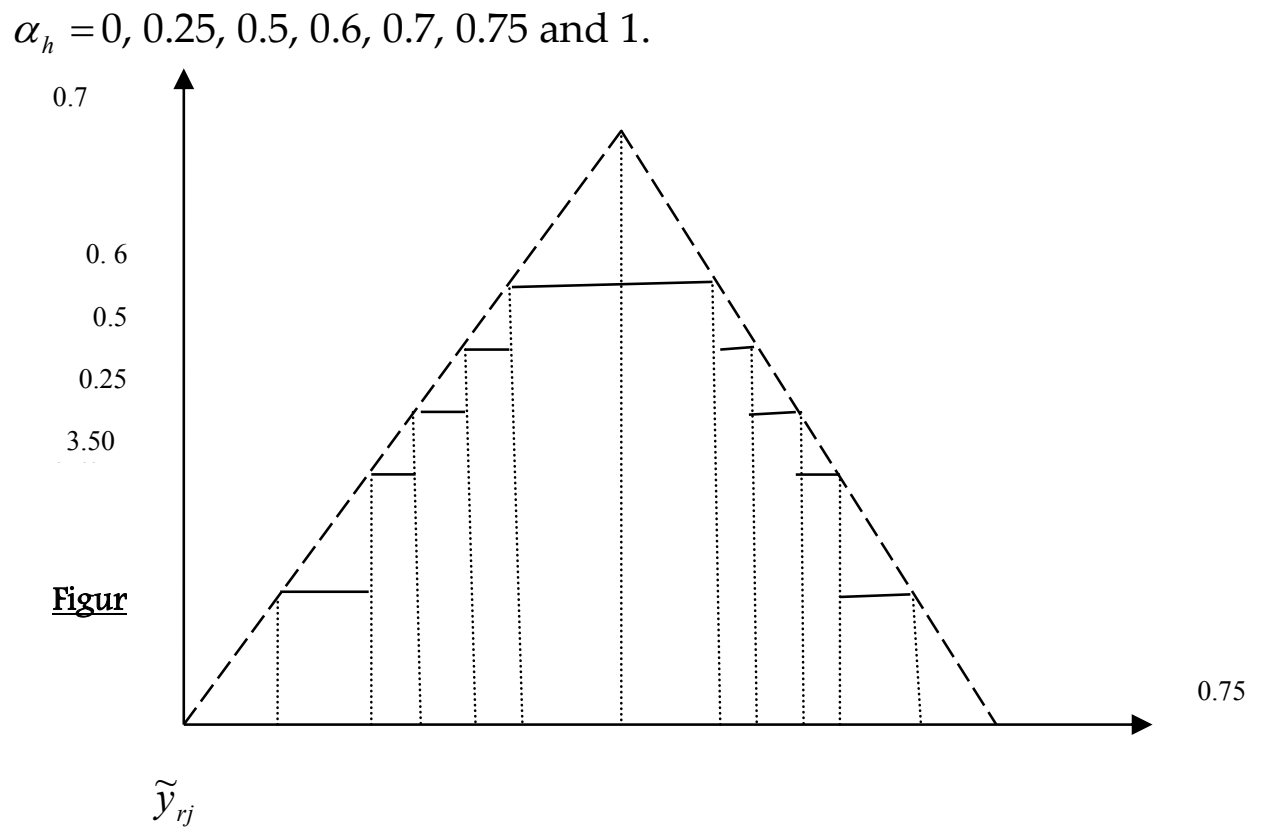


It should be noted that increasing the numbers of local $\alpha$-levels will result more precise measure of efficiency. For example when measuring efficiency of $\mathrm{DMU}_{1}$ if $\alpha$-levels are $[0,0.5,0.75,1]$ we obtain efficiency score of " 0.909 ", while if $\alpha$-levels are $[0,0.25,0.5,0.7,0.75,1]$ we obtain efficiency score of 0.903 which is a more accurate estimation of efficiency. Comparing these results with Table (2) it is clear that our proposed approach is given a better estimation of the efficiency scores when data are in the form of interval values. One drawback of the proposed model is longer computational calculation; however this is not a major issue with development of high-speed computers.

\section{Conclusion}

In evaluating DMUs in Fuzzy DEA there are four traditional approaches; the fuzzy ranking approach, the defuzzification approach, the tolerance approach and the $\alpha$-cut based approach. Each of these methods has its advantages and drawbacks in the way they treat uncertain data in DEA models. Perhaps due to its simplicity, the $\alpha$-cut based approach is frequently used by DEA scholars. As result of simplicity in this approach we will lose a lot of uncertainty information. This paper proposed an alternative fuzzy DEA technique for measuring efficiency of decision making units under fuzzy environment using local $\alpha$-level concept and linear programming problem. The numerical example showed a better estimation of efficiency when using the proposed model. The final model presented is a multi-objective programming, its transformation to a linear programming and developing an efficient algorithm for large scale problems are subjects for future development.

\section{References}

[1] I. Alp, Possibilistic Data Envelopment Analysis, Mathematical and Computational Applications. 7 (2002) 1: 5-14.

[2] B. Asady, A. Zendehnam, Ranking fuzzy numbers by distance minimization, Applied Mathematical Modeling. 31 (2007): 2589-98.

[3] A. Charnes, W.W. Cooper, E. Rhodes, Measuring the efficiency of decision making units, European Journal of Operation Research. 2(1978): 429-44.

[4] W. D. Cook, L. Seiford, Data envelopment analysis (DEA) - Thirty years on, European Journal of Operational Research, (2008) in press: doi:10.1016/j.ejor.2008.01.032

[5] A. Emrouznejad, An alternative DEA measure: A case of OCED countries, Applied Economic Letters. 10 (2003): 779-782. 
[6] A. Emrouznejad, E. Thanassoulis, A mathematical model for dynamic efficiency using data envelopment analysis, Journal of Applied Mathematics and Computation. 160 (2005) 2: 363-378.

[7] A. Emrouznejad, E. Thanassoulis, Measurement of productivity index with dynamic DEA, International Journal of Operational Research (2010), in press.

[8] A. Emrouznejad, Measurement efficiency and productivity in SAS/OR, Journal of Computers and Operation Research. 32 (2005) 7: 1665-1683.

[9] A. Emrouznejad, B. Parker, G. Tavares, Evaluation of research in efficiency and productivity: A survey and analysis of the first 30 years of scholarly literature in DEA, Socio-Economic Planning Sciences. 42 (2008) 3: 151-157.

[10] T. Entani, Y. Maeda, H. Tanaka, Dual models of interval DEA and its extension to interval data, European Journal of Operational Research. 136 ( 2002) 1: 32-45.

[11] R. E. Esteuer, Multiple criteria optimizing: theory, computation and application, John Wiley E Sons, Inc 1976.

[12] K. Field, A. Emrouznejad, Measuring the Performance of Neonatal Care Units In Scotland, Journal of Medical Systems. 27 (2003) 4: 315-324.

[13] P. Guo, H. Tanaka, Fuzzy DEA: A perceptual evaluation method." Fuzzy Sets and Systems. 119 (2001) 1: 149-160.

[14] J. L. Hougaard, A simple approximation of productivity scores of fuzzy production plans, Fuzzy Sets and Systems. 152 (2005) 3: 455-465.

[15] M. Inuiguchi and T. Tanino, Data Envelopment Analysis with Fuzzy Input-Output Data, Y. Haimes and R. E. Steuer: Research and Practice in Multiple Criteria Decision Making, 296-307, Springer (2000).

[16] C. Kao, S. T. Liu, Fuzzy efficiency measures in Data Envelopment Analysis, Fuzzy Sets and Systems. 113 (2000): 427-437.

[17] J. M. Kirigia, A. Emrouznejad, R. G. Vaz, H. Bastiene, J. Padayachy, A comparative assessment of performance and productivity of health centres in Seychelles, International Journal of Productivity \& Performance Management. 57 (2008) 1.

[18] T. Leon, V. Liern, J. L. Ruiz, I. Sirvent, A fuzzy mathematical programming approach to the assessment of efficiency with DEA models, Fuzzy Sets and Systems. 139 (2003): 2 40719.

[19] S. Lertworasirikul, S. C. Fang, A. Jeffrey, J. A. Joines, H. L. W. Nuttle, Fuzzy Data Envelopment Analysis (DEA): a possibility approach, Fuzzy Sets and Systems. 139 (2003): 2 379-94.

[20] S. Lertworasirikul, Fuzzy Data Envelopment Analysis for Supply Chain Modeling and Analysis, Dissertation Proposal in Industrial Engineering, North Carolina State University. 2001.

[21] S. Lertworasirikul, S. C. Fang, H. L. W. Nuttle, J. A. Joines, Fuzzy BCC model for data envelopment analysis, Fuzzy Optimization and Decision Making 2 (2003): 337-358. 
[22] Y. Meada, T. Entani, H. Tanaka, Fuzzy DEA with interval efficiency, Proceedings of 6th European Congress on Intelligent Techniques and Soft Computing. EUFIT '98. Aachen, Germany, Verlag Mainz. 2 (1998): 1067-71.

[23] R. Mulwa, A. Emrouznejad, L. Muhammad, Economic efficiency of smallholder maize producers in western Kenya: A DEA meta-frontier analysis, International Journal of Operational Research. 6 (2008): 3 In Press.

[24] R. Mulwa, A. Emrouznejad, F. M. Murithi, Impact of liberalization on efficiency and productivity of sugar industry: The case of Mumias sugar company in Kenya, Journal of Economic Studies. 2008 in Press.

[25] S. Saati Mohtadi, S. A. Memariani, G. R. Jahanshahloo, Efficiency analysis and ranking of DMUs with fuzzy data, Fuzzy Optimization and Decision Making. 1 (2002) 255-267.

[26] J. K. Sengupta, A fuzzy systems approach in Data Envelopment Analysis, Computers and Mathematics with Applications. 24 (1992) 8: 259-66.

[27] M. Zerafat Angiz, A. Emrouznejad, A. Mustafa, A. Rashidi Komijan, Selecting the most preferable alternatives in a group decision making problem using DEA, Expert Systems with Applications. 36 (2009) 9599-9602.

[28] M. Zerafat Angiz, A. Emrouznejad, A. Mustafa, A. Fuzzy assessment of performance of a decision making units using DEA: A non-radial approach, Expert Systems with Applications. 37 (2010) $5153-5157$.

[29] M. Zerafat Angiz, A. Emrouznejad, A. Mustafa, A. S. al-Eraqi (2010) Aggregating Preference Ranking with Fuzzy Data Envelopment Analysis, Knowledge-Based Systems, In Press.

[30] H. J. Zimmermann Fuzzy set theory and its applications, Kluwer Academic Publishers. 2001. 